REMLATE DE MLALIES

Campinas-SP, v. 38, n. 1, pp. 414-436, jan./jun. 2018

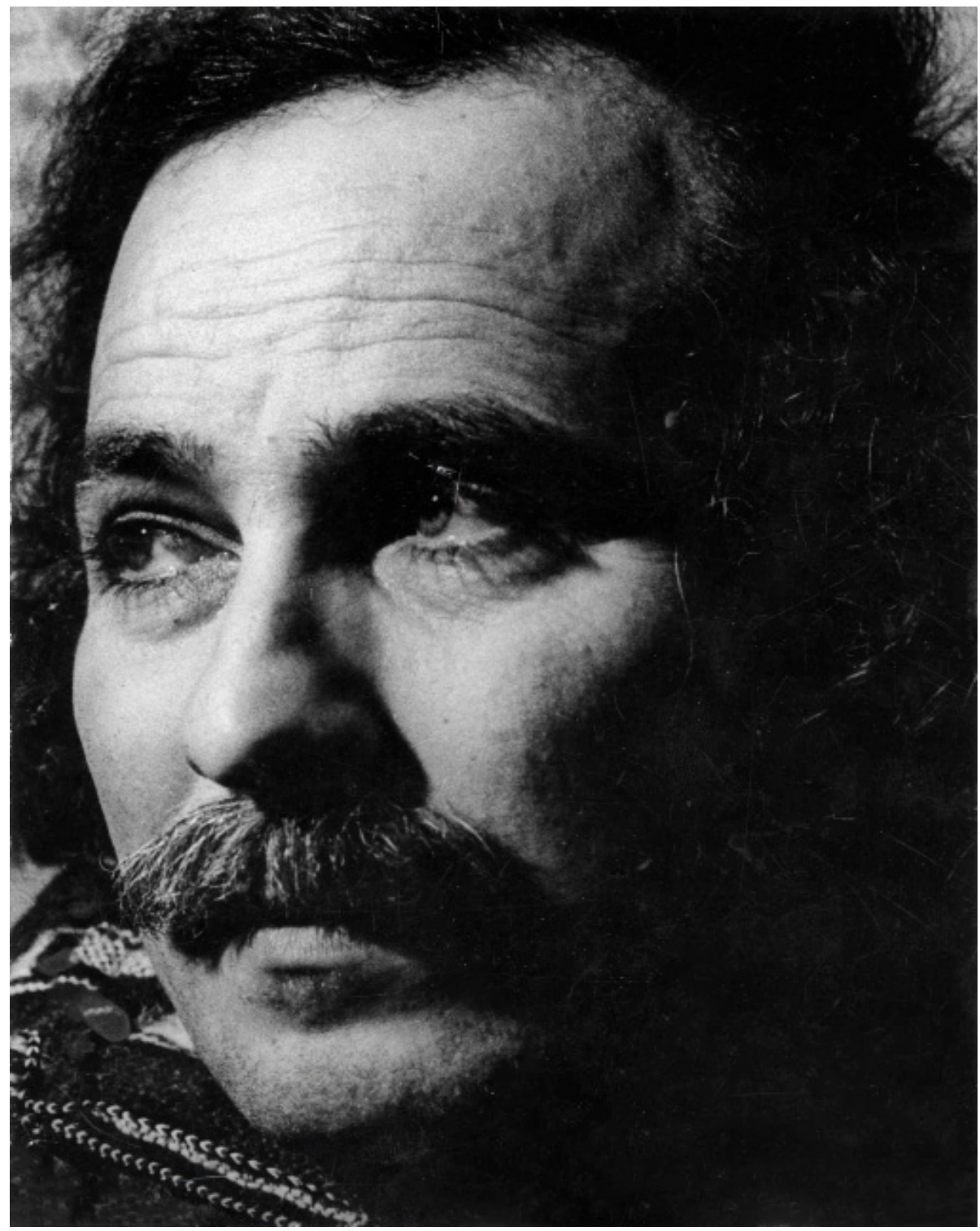




\title{
Virgílio de lemos, Poeta do Oceano ÍNDICO
}

\author{
Jessica Falconi
}

Nascido em 1929 na Ilha do Ibo, Virgílio de Lemos foi uma figura de destaque dos círculos intelectuais da colónia de Moçambique das décadas de 1940 e 1950. Autor de uma vasta obra poética, assinada por vários heterónimos - Duarte Galvão, Bruno dos Reis, Lee-Li Yang - colaborou com publicações periódicas diversas, como o Jornal da Mocidade Portuguesa, Itinerário, O Brado Africano, A Voz de Moçambique, etc.

Em 1952 fundou a rivista Msaho, um projeto cultural que só teve um número, mas que marcou a história da literatura em Moçambique. Esboçava-se, em Msaho, uma pluralidade de vertentes temáticas e estéticas que é hoje reconhecida pela crítica como caraterística fundadora da poesia moçambicana (MENDONÇA, 2008; NOA, 2008; LEITE, 2003 e 2008). Relembrando o projeto da revista, Virgílio de Lemos afirmava, numa entrevista recolhida por Carmen Tindó Ribeiro Secco: «Desejávamos alargar os horizontes culturais de Moçambique dos anos 40-50. Essa resistência tinha de se concretizar e, na época, isso só era possível através das páginas literárias dos jornais quotidianos, dos semanários» (SECCO, 1999:150)

Apesar de pertencer à elite colonial, Virgílio de Lemos foi alvo da PIDE, tendo sido preso pelas suas atividades de denúncia das injustiças 
do sistema colonial. Em 1963, exila-se na França, onde haveria de ficar até à morte, a 6 de dezembro de 2013.

No prefácio ao livro "Para Fazer um Mar", o poeta moçambicano Luís Carlos Patraquim situa pela primeira vez a poesia de Virgílio de Lemos no quadro mais amplo da poesia do Oceano Î́ndico (PATRAQUIM, 2001), convidando-nos a uma viagem pela rica imagética insular criada pelo poeta e pelos diálogos transoceânicos por ele estabelecidos. Para Virgílio de Lemos, as ilhas moçambicanas são lugares paradigmáticos para se 'provincianizar a Europa', abdicando de qualquer noção identitária fundada na pureza e no essencialismo (FALCONI, 2013).

Como que dialogando com o posicionamento epistemológico invocado e assumido por Isabel Hofmeyr no artigo "O Atlântico Negro encontra o Oceano Índico”, a palavra poética de Virgílio de Lemos olha ora para a construção cultural da negritude, ora para as "culturas do Índico", ecoando o imaginário criado por poetas como Jean-Joseph Rabearivelo, Édouard Maunick, Jacques Rabemananjara, entre outros.

A atividade poética e cultural de Virgílio de Lemos, bem como a sua trajetória pessoal e intelectual, representam um ponto de partida possível para se olhar e repensar o Oceano Índico a partir de Moçambique e das narrativas em língua portuguesa. Inúmeros são os poemas de Virgílio de Lemos dedicados às águas e às ilhas do Índico, destacando-se os livros Ilha de Moçambique. A língua é o exílio do que sonhas (1999) e Para Fazer um Mar (2001).

Os documentos que se publicam foram-me cedidos pelo autor durante uma estadia dele em Nápoles, em 2007. Acompanhado pelo amigo e jornalista francês Thierry Perret - responsável pela paginação e impressão do caderno L'ecart du temps - Virgílio de Lemos participou, na altura, numa jornada de Culturas Lusófonas organizada na cidade de Salerno.

Trata-se de um pequeno fragmento do vasto arquivo do autor cuja publicação pretende contribuir para recuperar da sua escrita à luz de novas cartografias da poesia do Oceano Índico. 


\section{1- CADERNO IMPRESSO}

Título: L'ecart du temps

13 páginas digitalizadas

O caderno contém duas secções de poemas: a primeira, intitulada "L'ecart du temps", data de 2003; a segunda, intitulada "Impressions des Indes", data de 1991.

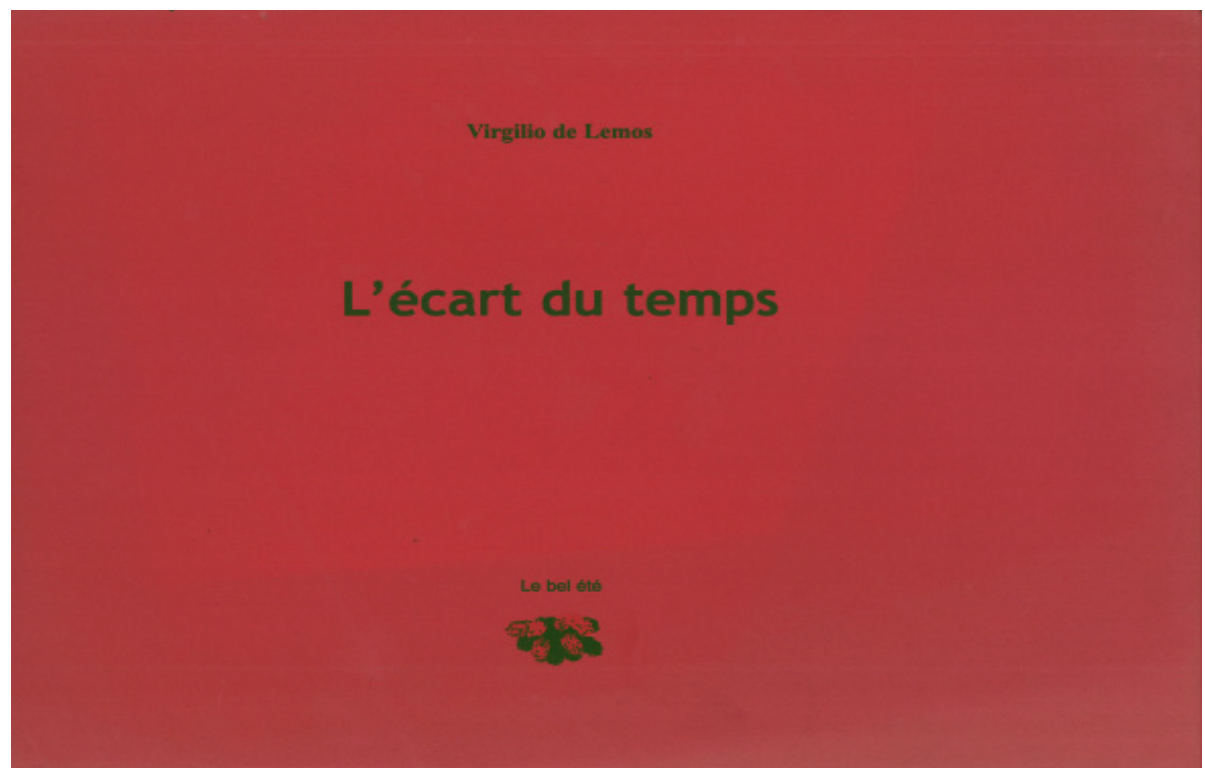


Caderno impresso - 418

Virgilie de Lemos

\section{L'écart du temps}

(2003)

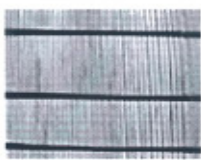

suivi de

Impressions des Indes (1991)

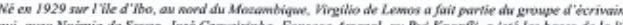

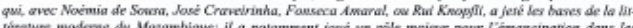

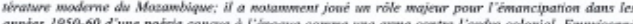

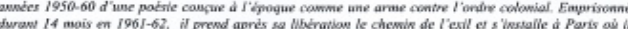

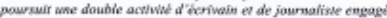

Vingiwio de Lemos écrit en frumcais depuis 1967, a ne cessera plus de composer dans les dena langues

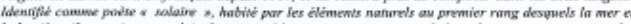

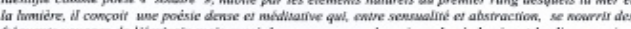

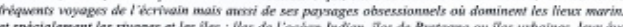
作

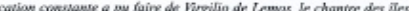

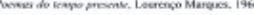

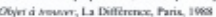

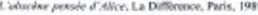

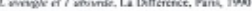

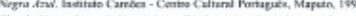

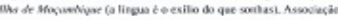

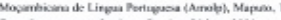

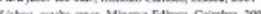

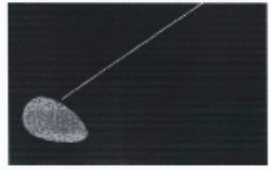


Remate de Males, Campinas-SP, v. 38, n. 1, pp. 414-436, jan./jun. 2018 - 419

\begin{tabular}{|c|c|}
\hline \multicolumn{2}{|l|}{$\begin{array}{l}\text { L'écart du temps } \\
\text { (2003) }\end{array}$} \\
\hline \multicolumn{2}{|l|}{ L. } \\
\hline \multicolumn{2}{|l|}{ Les candes de ton are } \\
\hline $\begin{array}{l}\text { Ton violion d'Ingres } \\
\text { Ne s'eniverent pas de ter mots }\end{array}$ & 4. \\
\hline Ni de ta voix & Ne te force pas. Ne force pas \\
\hline Dans le volcan de l'ettre & La tentation de la langue \\
\hline Le soir se nourrit de musique & Le langage se veut nussi Trame \\
\hline Mktaphore de I'indicible. & $\begin{array}{l}\text { Du lyrisme } \\
\text { La réplique de l'idece. }\end{array}$ \\
\hline \multicolumn{2}{|l|}{2.} \\
\hline Les couleurs les lignes les marges & 5. \\
\hline Les sons disent le regard & L'étre est un volcan \\
\hline De la poésie. Le corps de l'ãme. & Qui fremit i l'art \\
\hline Ta voix. & Du sang de l'imelligence \\
\hline & $\begin{array}{l}\text { De la spiritualité qui vibre. } \\
\text { Le langage ditrape of }\end{array}$ \\
\hline 3. & Exprime la négation de la forme \\
\hline \multirow{2}{*}{\multicolumn{2}{|c|}{$\begin{array}{l}\text { La poesie refuse le langage } \\
\text { lsolece }\end{array}$}} \\
\hline & \\
\hline \multicolumn{2}{|l|}{ de toen corps } \\
\hline \multicolumn{2}{|l|}{$\begin{array}{l}\text { elle ne retient que } \\
\text { le crissement de tes os }\end{array}$} \\
\hline \multicolumn{2}{|l|}{$\begin{array}{l}\text { le crissement de tes } 06 \\
\text { la poussictre }\end{array}$} \\
\hline \multicolumn{2}{|l|}{$\begin{array}{l}\text { qui s'evade de } \\
\text { tes murmures }\end{array}$} \\
\hline le feu du regard. & \\
\hline
\end{tabular}
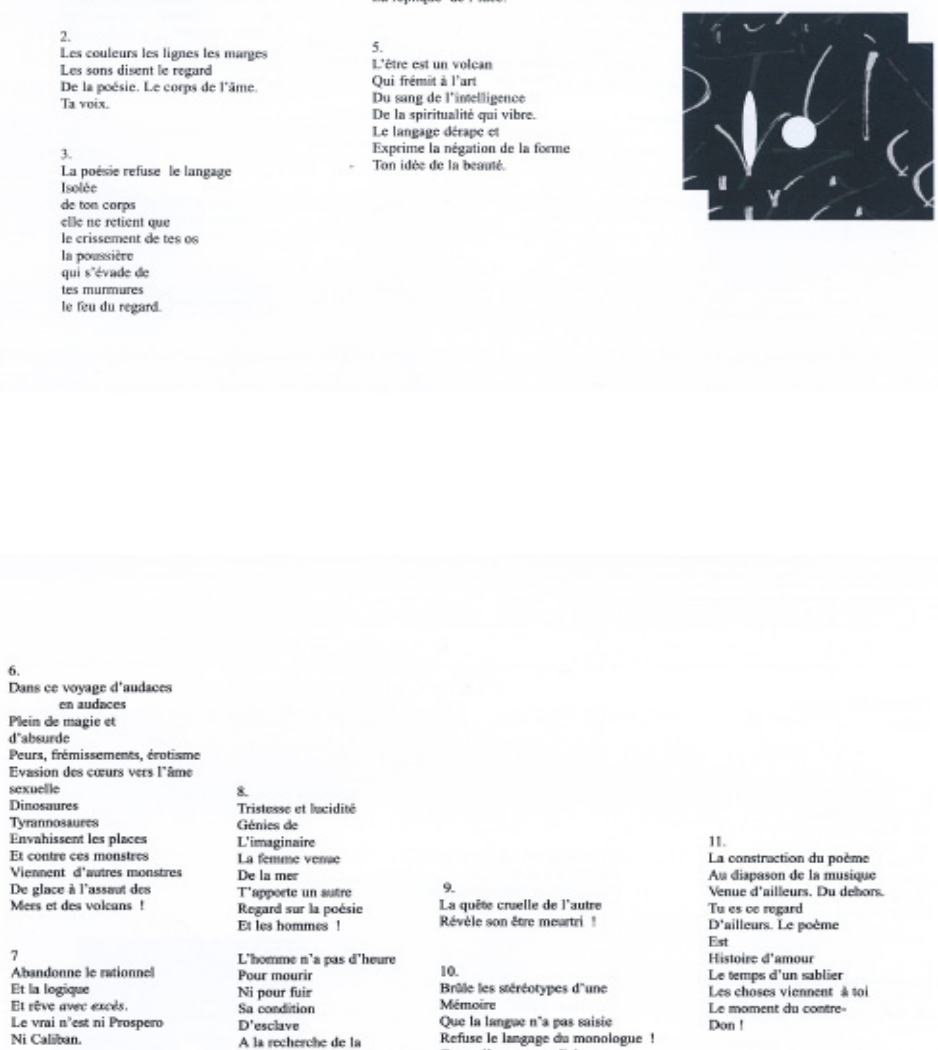

Tristesse et lacidite

Génies de

Limaginaire

De la mer

T'sppote un witre

Et les hommes !

9

La quete cruelle de l'autre

11.

Revele san cere mearti !

L'hoemme n'a pas d'heure

Pour mourir

$\mathrm{Ni}$ pour fuir

10.

Bralle les stertocypes d'une

$\begin{array}{ll}\text { Sa condition } & \text { Mémoire } \\ \text { D'eschive } & \text { Que la langewe n"a pas saisie } \\ \text { A la rochercte de la } & \text { Refuse le langage da monalogue }\end{array}$

$\begin{array}{ll}\text { A la rocherchte de la } & \text { Refuse le langage da manolo } \\ \text { Liberte ! } & \text { Owwre lespace au dialogue }\end{array}$

La constraction du poème

Ava diapason de la musique

Tu es co rogard

D'ailleur. Le poetm

Histoire d'amour

Le temps d'un sablier

Les choses viennent at toi

Don 
12. L'osmose handic Entre la mise ch sctene Loppressant

Evite les embiches

Cite ton propre langage.

La force du style

Fis ke clin d'reil

De tan ironic?

13.

La magie et le

Discours de l' tre

Crete ta propre masique

Refus des règles et

Rythmes qui chang
Au gre du soir !

II observe le fond de la mer Derrictre ses lunettes de coraleur Oublie ses desiltusions ses

Désenchanterments

Ses chiméres.

Son nouvel espace sous-marin.

20.

Le sad da Sud

Oavre ses portes imvisibles

Les portes sanvages

Le gúnie sous-marin de la mer

Crete des espaces nouveaux

Dans son labyrinthe it n'y a

De place que pour la marge.

Qui sumuit d"ailleurs

Voulu le suivre?

A faire le voyago
Péneterer le ciel

Et la mer de glace ?

22.

D'inconnu

De fenters entr' ouverts

De lienes

Sans frein récits de woyages a

Réinventer des fortets

Des méraphores maufragces !
14

Un pas vers les couleur:

Pas nouvewa des soms

Intermittente magie de

L'inconscient.

Fragnentation des mot:

Ton désir s'approprie

Ellipese des mots en retrait

Dans l'écravement

Des sensations et

Des os!

15.

Aucun regard

Sur mon enflance

Ne montre la rupture

Entre moi et mon miliet

Er mon père

Moi-méme !
Pas de triemphe des hommes Sur I'Histoire. Loin de to

Diswours dominatcur

Ma poesie peut-elle

L'tie od son regand

Avu le jour

A vu le jour

Le désastre de l'homme?

Ruptures obscures

Transparences

Seule la poésie elue

Par son tre profond.

La visise du temps

L'éloigne de
Pexché sur la mer du Cap

Des Templetes

Son regard s conve an bruit des

Tempetes

Aux cris des oiseana

Aur blocs de neige

De l'Antaretique !
23.

Chaque podete isterit son

Vyyage

Crée le Sud du monde

Le sotnario d'un film

Qui repand

Aux aspirations
Ne latsse pas somber

Laventure, Verne et London

Taccompagnem

D. Quixote es Kan

Omar Khayyim

Habivent I'histoire

Du poete !

26.
*ftre $*$ l'essence de ton âme

C'est bien être ta vie

De saing

Les échafaudages

Du corps intérieu

Enignes du corps et

Tes mots

Tu emtres dans le lieu

Dams le lieu were

Qui e'exipe pas

De nouvelles guernes 
Remate de Males, Campinas-SP, v. 38, n. 1, pp. 414-436, jan./jun. 2018 - 421
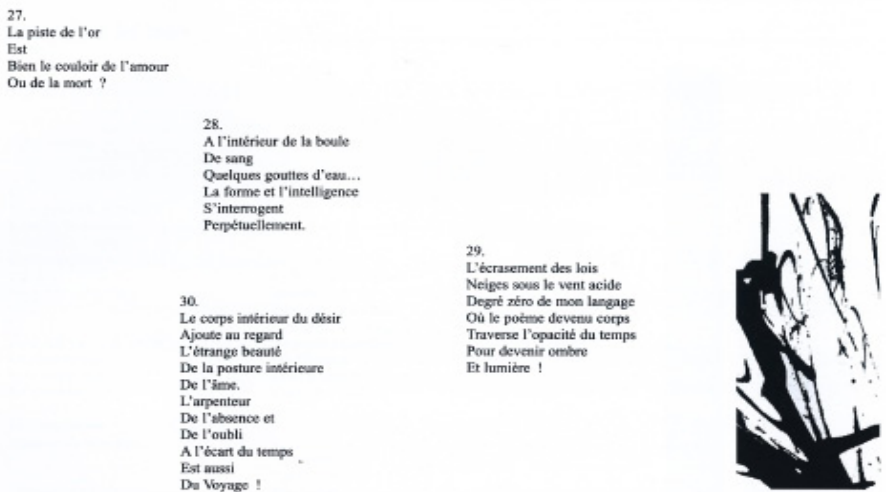

Impressions des Indes

(1991)

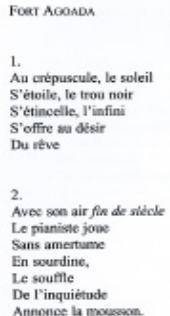

3. La beauté est artifice

Sublimation du deuil.

Prisonnier de l'tenigme

Par listemel

Brume subtile

Corps de l'illusioe

4.

La poesie

Guette la briblh

De l'infini

Ala seut du non-dis.

L'humilite du regard

Fait la splendese et I"humiline

Du genie.

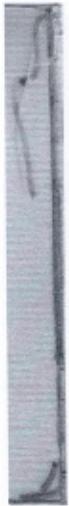


Remate de Males, Campinas-SP, v. 38, n. 1, pp. 414-436, jan./jun. 2018 - 423

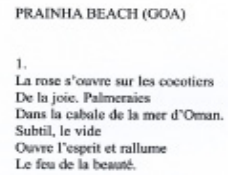




\section{2 - CAderno de AUtógrafo}

Título: A exuberância do nada

4 folhas de papel azul A4 dobradas

9 páginas digitalizadas

\section{Poemas}

1-" A exuberância do nada" - poema publicado em Ilha de Moçambique. A língua é o exílio do que sonhas, com pequenas variações (1999:94).

2 - E no grego silêncio das coisas ... - poema publicado em A Dimensão do Desejo (2012:15).

Os restantes poemas provavelmente são inéditos, não tendo sido encontrados nas edições consultadas:

3 - «Memórias da ilha» 1

4 - «Memórias da ilha 2

5 - «A vida não é o que parece»

6 - O segredo das Misericórdias....

7 - «Julieta, meu amor, minha ironia».

8 - «Arde a barca no teu corpo»

Edições consultadas:

IIha de Moçambique. A língua é o exílio do que sonhas, Maputo, AMOLP, 1999. 
Eroticus Moçambicanus: breve antologia da poesia escrita em Moçambique (1944/1963). Org. Carmen Tindó Secco, Rio de Janeiro, Nova Fronteira/UFRJ,1999.

Para fazer um mar, Maputo, Instituto Camões, 2001.

A Invenção das Ilhas, Org. António Cabrita, Maputo, Escola Portuguesa de Moçambique - EPM; Centro de Ensino e Língua Portuguesa- CELP, 2009.

Jogos de prazer, org. Ana Mafalda Leite, Lisboa, Imprensa NacionalCasa da Moeda, 2010.

A Dimensão do Desejo, org. Matteo Angius, Maputo, AMOLP, 2012. 
Caderno de autógrafo - 426

A exuberincia do nade

(cha de merambius)

VIRGILI O TE LEMOS 
Remate de Males, Campinas-SP, v. 38, n. 1, pp. 414-436, jan./jun. 2018 - 427

A exuberincia do nada

Femininos são os sthas je nos sham ua pervensäo dos sentidos e da glánie.

Femumina éa ilusas e a retóvica suitil da osteutecad.

Fenulina éa busca do saber e da verdade / we nos escape a enfacudora miragen da persuasão.

A tautasia emprestas singular - Paceo do lumor. a parádoxal irmia. do Nada.

sejundo a verve e avoj dos dialogautes, incham ou mirram os Tounates do diabo, feminivos.

itha de manbive/1957 virglio e emos? 
Caderno de autógrafo - 428

E no grego silaincio das coisas, deste secreto vulcäo, panto may use ocho iviedativel, menor me vego, neais fundo ure sinto.

itha de muxacultip/1959 visitiode temol: 
Remate de Males, Campinas-SP, v. 38, n. 1, pp. 414-436, jan./jun. 2018 - 429

Memónias da ilha

1.

Na espenca vives

de (ue o áceantá sega agorn eapui,

somb real

entic : gesto

e a ricajem,

entre o som

e a liij) vajem,

na cadoncia nobre

de prem sabe

ue a ride

presa por un fis é

frajil corda

lue se agita

e uos mata.

illea de micaurb/e / 1552

virgitio dénos. 
Caderno de autógrafo - 430

Yenuorias da ille

2.

Sussuras-me pase as ouvido

palarvas pe ma/ ouco

sous que vibrem destro de mion

notas muricais $t \geq 1$ he 3 da somora visã.

de (ueme reinventa

- Amer,

imagers no esplecedor

de queme

nada recusa a emocid.

illate morantige/1952 vighio ar cemol:- 
Remate de Males, Campinas-SP, v. 38, n. 1, pp. 414-436, jan./jun. 2018 - 431

A VIDA NF̈O É O PUE PARECE ( nem secupre é a licijua luem mellor a iraduz) (A ARISBóteles)

No Teu cabedal de saber e ternura - erpace da daidiva é seer limites. Do jue trazes, porpur o tazes, coises feitos. navegam eutre a incertga eo absurto. No ten secreto rio exitre tulgores e tabua de valores, funcal dos actos, never sempre éa lingra 'uemu trellora a. Na fore, des cretrínios derliza avela.

A ájua brota pelas eivas, ditada pelo expinto, secure éa drlidar do canto, docurre malicia, feitos.

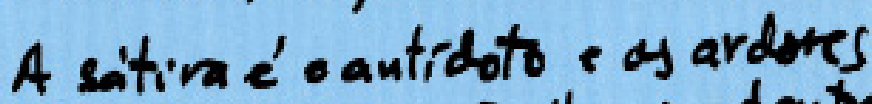
das almas /ve ze uas ollom por deutro.

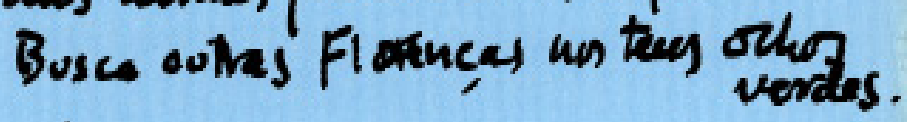
itha de mos/1857

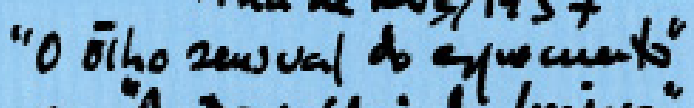
ar "A rafédeci dal linjua" 
Caderno de autógrafo - 432

0 segrëdo das Mexiricardias navegore enth Afuicas e Brasis, entre Brases Indias, entre $0 R_{\text {is }}$ a Baća eo hang do Ja Paulo. - Lavp de neutonas e repédes da ressurvicas da icha da ressurecáf dos escravos. das donase sás, arabies, carnes edreias isodis.

Enyato os encravss ajavas e maknas suferzm - Dr salkodas mukes na cavea de acucar e nos arrozais

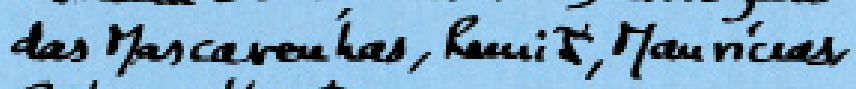
Cuba e Harti. as mais belas ads bescerten. molkers recinventam o acuor $e$ a vida nos sulteuatos execados d'Ansia e das Goas dorrades we os sonkos multifhicam. of sepredos da colica e das parxoer evrom ainda, almá de namfrapuar Tesoniras fue o mandevora lectancente.

itha de unveank/up/1956 layg do Str Parilo

(Cnrersa can Cortesar Casrumin, con Auilcar. Fermeses Hanuel Bavreto) 
Remate de Males, Campinas-SP, v. 38, n. 1, pp. 414-436, jan./jun. 2018 - 433

Julieta, men amor, minka irsia (o gue tazer de ten conscal)

Yau corpo todo se embriaga de tens gostos palavios, tensenleros, tie máfica is, desejo un sas suspiros, cuctro Além, sipplices que neu corragl abraca. Yeu compo todo de extasia e en canto ene grogo, ew perses, a luy da tha gracea lue rie chove, nem jura nem promete, e fay da alejvia butono e Pnincerera. 0 mundo d belo, entre kagrimas e prazer evra tua sede de eterno, in/uieta e seduTora, improvijas can caej de emsalar. Imitas a pauxes, o canto do vuláio ao mear, a verito. Debruças. Te as balcōoo da utopia, es deuse e desejo, min ka sombre, fontasia.

Tha do ibo/1957 virgilio de Lemos: 
Caderno de autógrafo - 434

ARde a barca no teo corpo

Ibor sando mares na vertifen froipha de quevi intengrdades busca, pengo en ti, e excrevo-te nesta lonad toite que $r i$, agreste luz.

Itotgando cla ridades e sombras, breves teusēen e termuras, te revejo eutre velas e ventos jue se crugam indiprentes a melanislo da almax.

Ibor sando, nar adivin hus (vourto em min sistes, na, purga dos gestos e gritas. nos mur múnios in rentades da beleza. Felicidales, ua in certega do $\infty$ évago e traf́l, unas intenso', (udito a darca no teu corpo arde e o mar chora.

icha ds ibo/1957 rors julieta \%, muther swakili-meativa. 
Remate de Males, Campinas-SP, v. 38, n. 1, pp. 414-436, jan./jun. 2018 - 435

\section{3 - Diapositiva}

1 imagem digitalizada

Rosto de mulher, provavelmente da Ilha de Moçambique.

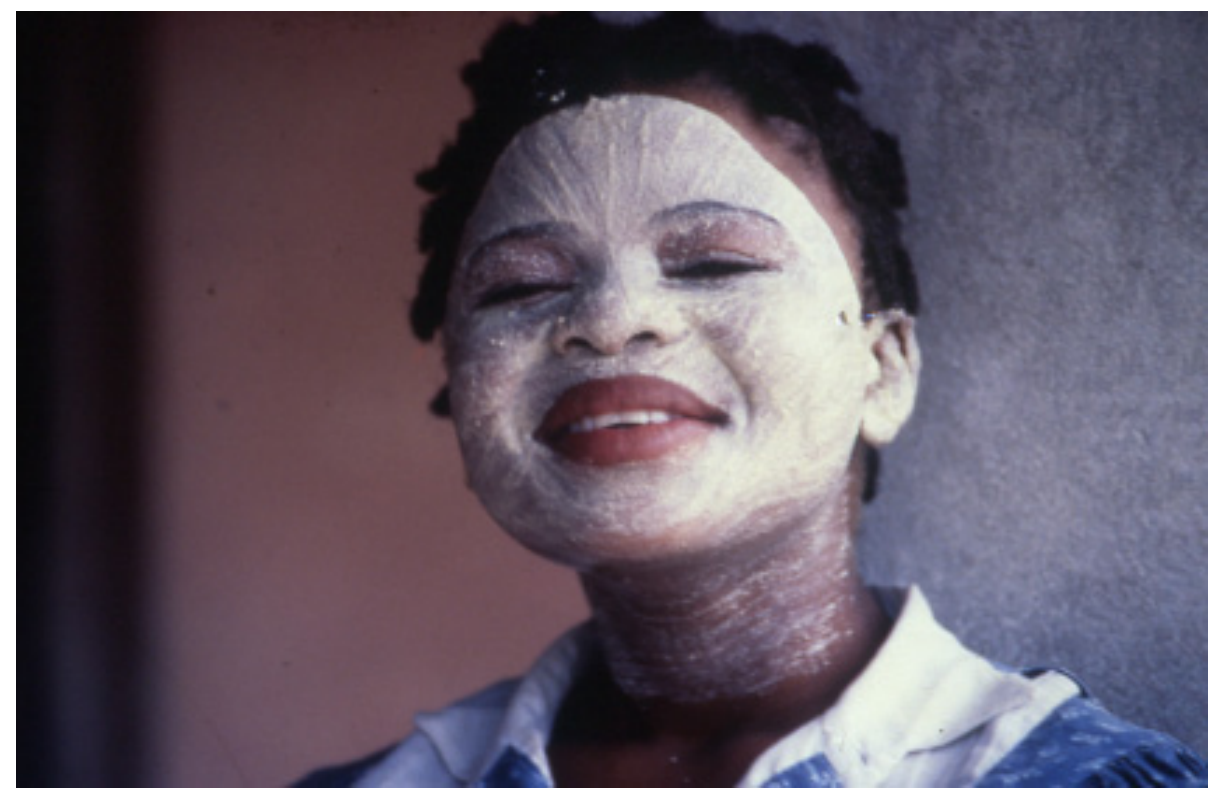




\section{REFERÊNCIAS BIBLIOGRAFICAS}

FALCONI, Jessica (2013) "Para fazer um mar. Literatura moçambicana e Oceano Índico", Diacritica v. 27, 77-92.

LEITE, Ana Mafalda (2003), Literaturas africanas e formulações pós-coloniais. Lisboa: Colibri.

LEITE, Ana Mafalda (2008) “Tópicos para uma História da literatura Moçambicana”. In: Moçambique das Palavras Escritas (org. Margarida Calafate Ribeiro e Maria Paula Meneses), Porto, Edições Afrontamento, p.47-76.

LEMOS, Virgílio de (1999) Ilha de Moçambique. A língua é o exílio do que sonhas. Maputo. Associação Moçambicana de Língua Portuguesa.

LEMOS, Virgílio de (2001) Para Fazer um Mar. Lisboa. Instituto Camões.

MENDONÇA, Fátima (2008), "Literaturas emergentes: identidades e cânones". In: Margarida Calafate Ribeiro \& Maria Paula Meneses (orgs.) Moçambique: das palavras escritas. Porto: Afrontamento, 19-33.

NOA, Francisco (2008) "Literatura moçambicana: Os trilhos e as margens”. In: Margarida Calafate Ribeiro \& Maria Paula Meneses (orgs.) Moçambique: das palavras escritas. Porto: Afrontamento, 35-46.

PATRAQUIM, Luís Carlos (2001) “O senhor das ilhas” in Virgílio de Lemos, Para Fazer um Mar. Lisboa: Instituto Camões, 7-9.

SECCO, Carmen Tindó Ribeiro (org.) (1999) Eroticus Moçambicanus: breve antologia da poesia escrita em Moçambique, 1944-1963. Rio de Janeiro: Nova Fronteira. 